\title{
Design and Implementation of Census Data Collection System using PDA
}

\author{
A.Vijayaraj \\ Associate Professor, IT Department \\ Saveetha Engineering College, Thandalam \\ Chennai-602 105 Tamil Nadu, India.
}

\author{
P.DineshKumar \\ Asst. Professor, IT Department \\ Dr.M.G.R University,Maduravoyal \\ Chennai- 600 095, Tamilnadu,
}

\begin{abstract}
This paper mainly concentrates on improving the efficiency of census data collection. The usual census data collection system involves an authorized enumerator, who collects the census data manually with paper and pen. This method has been a lot of time consuming and painstaking one. So in today's world of data communication a client tool for a portable system becomes important to access and update a database

Innovative thinking helps us to improve the existing system .So we thought the finest solution for this handicap would be using a PDA. But such a client tool is yet to be developed. We have designed and developed such a client tool with PDA .Our idea is to implement this by providing a PDA containing the census application to every enumerator authorized by government through which they can collect census data and update the collected data to the census database thus saving time and money.
\end{abstract}

Keywords: PDA, Census application, Data Collection, Enumerator

\section{INTRODUCTION}

Information Technology is a wonderful finding of present day's technological development. This is in fact influencing even our day to day activities. When we come to some specialized forms of work, Information Technology's part is even more important and it comes handy. For our paper too it is playing a major role. In our paper we are using Information Technology methods to collect census data. The term Census implies that the each individual and each set of individual characters are enumerated separately and that the characteristics thereof separately recorded. Census organization has experimented with new IT innovations since the beginning. Technology is required particularly for data capture/processing mainly due to large volume and for speedier tabulation \& release of Census results. This is made possible by using PDA as a client tool which contains the census data collection application in it. Registrar General and Census Commissioner of India under the Home Ministry authorize state census commissioners to collect census data through authorized personnel. The Enumerators are selected and adequate training is given to them in using the equipment. The PDA is then given to the trained enumerators who are the personnel authorized by the government. The enumerator collects census data and updates the census database. This increases the efficiency of the work and a great time is saved. The enumerator is allotted a particular residential area for each day and within a stipulated time they should complete their allotted area. They obtain help from revenue department to list the census houses. The obtained census data is commonly is used for research, business marketing, and for preparing the five-year plan.

\begin{abstract}
II. LITERATURE REVIEW
Existing System: In Indian census data collection, generally these steps are to be followed. Identifying the houses i.e., dwelling places and places that are usually used for living like sheds that are used by nomads. This is the first step. Then the enumerator prepares the house list. Once the house list is prepared the enumerator prepares sketches of blocks of houses that give the primary information about the type of houses and facilities that are being enjoyed by the population in that area. The house list acts as the frame work for the next step of enumeration. Enumerator reports to supervisor and supervisor reports to the charge officer and charge officer reports to the area officer, area officer reports to zonal officer and zonal officer to census administrator of the state. The census administrators report to the Register general and census commissioner of India
\end{abstract}

Enumerator prepares notional maps (based on preliminary information) then the exact layout sketches of the blocks are prepared. Buildings and census houses are allocated numbers, based on which house census and establishment census are taken individually. Similarly all the numbering work is completed for the buildings and houses. With this information in hand they go to the census houses and collect data. The collected data would be used for analysis of the population in various aspects like finding population ratio, employment reports, and facilities available to people etc.

\section{Problem Description}

The above system is a very traditional method and involves many difficulties. Now-a-days in this fast world of technology it is very important to complete a work in a speedy manner. In the above mentioned method collecting data takes a long time, because enumerator has to manually fill in the census form then again sort out the data etc. So we need a system that segregates data as and when data is entered. Another difficulty in this method is the accuracy of data entered. In a census, age of people should be entered accurately because these details would be used in employment analysis etc. Efficiency is another issue; efficient data collection is needed because this data will be used for further analysis. Until now everything is done on paper. An authorized enumerator visits every single house and establishment and collects the complete information. This system has dual work of collecting the data on paper and then feeding it in computer. This also takes a vast amount of manpower into it. The information is subjected to change as it is taken as a written work on a paper.

\section{Proposed System}

The solution for the above mentioned for drawbacks is finding a device which has comparatively well equipped device (similar to laptop/PC) with network accessibility, consumes comparatively less 
power, easy to carry and able to work even while walking. The finest solution out in the market is PDA (Personal Digital Assistant). PDA is a device with high computation power.

A personal digital assistant (PDA), also known as a palmtop computer, is a mobile device which functions as a Personal Information Manager and connects to the internet The PDA has an electronic visual display enabling it to include a web browser, and also have audio capabilities, enabling them to be used as mobile phones or portable media players. PDAs can access the internet, intranets or extranets via Wi-Fi, or Wireless Wide Area Networks (WWANs). Many PDAs employ touch screen technology.

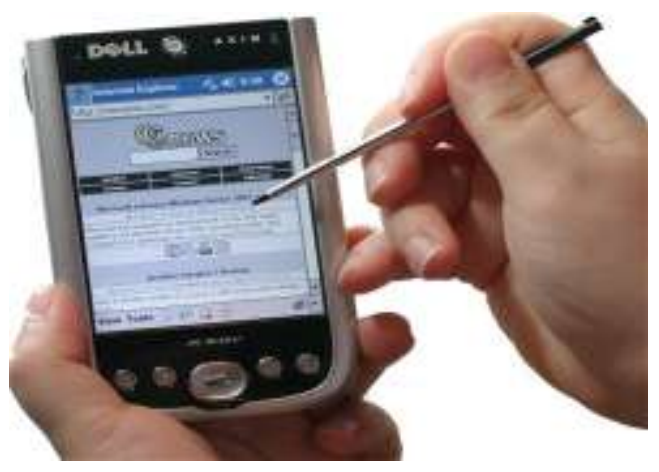

\section{Why PDA?}

A typical PDA has touch screen for entering data, a memory card slot for data storage and at least one of the following for connectivity: IrDA, Bluetooth and/or Wi-Fi. However, many PDAs (typically those used primarily as telephones) may not have a touch screen, using soft keys, a directional pad and either the numeric keypad or a thumb keyboard for input. Software typically required for a PDA includes an appointment calendar, a to-do list, an address book for contacts and some sort of note program. Connected PDAs also typically include E-mail and Web support.

The PDA is equipped with Microsoft's windows mobile operating system. The advantage of PDA is that we can develop customized applications for it like eVB(embedded Visual Basic), eVC++(embedded Visual $\mathrm{C}++)$ and Visual Studio.NET Compact framework. Another major advantage is its accessibility. (i.e.) data services like GPRS, Wi-Fi enable us to keep in touch with the servers. PDA (personal digital assistant) is a term for any small mobile hand-held device which provides both computing and information storage together with retrieval capabilities for personal and business use. It is often used for keeping schedule calendars and address book information handy. The term handheld is a synonym. Many people use the name of one of the popular PDA products as a generic term. Touch screen PDAs, including Windows Mobile devices, usually have a detachable stylus that can be used on the touch screen. Interaction is then done by tapping the screen to activate buttons or menu choices, and dragging the stylus to, for example, highlight. Text input is usually done as follows in a touch screen PDA. Using a virtual keyboard, where a keyboard is shown on the touch screen. Input is done by tapping letters on the screen. PDAs are now compatible with micro SD cards, which are physically much smaller than standard SD cards.

An important function of PDAs is synchronizing data with a PC. This allows up-to-date contact information stored on software such as Microsoft Outlook or ACT! to update the database on the PDA. The data synchronization ensures that the PDA has an accurate list of contacts, appointments and e-mail, allowing users to access the same information on the PDA as the host computer.

The synchronizing also prevents the loss of information stored on the device in case it is lost, stolen, or destroyed. Another advantage is that data input is usually a lot quicker on a PC, since text input via a touch screen is still not quite optimal. Transferring data to a PDA via the computer is therefore a lot quicker than having to manually input all data on the handheld device.

Most PDAs come with the ability to synchronize to a PC. This is done through synchronization software provided with the handheld, such as HotSync Manager, which comes with Palm OS handhelds, Microsoft ActiveSync for Windows XP and older operating systems, or Windows Mobile Device Center for Windows Vista, both of which sync with Microsoft Windows Mobile or Pocket PC devices.

These programs allow the PDA to be synchronized with a Personal information manager. This personal information manager may be an outside program or a proprietary program. For example, the BlackBerry PDA comes with the Desktop Manager program which can synchronize to both Microsoft Outlook and ACT!. Other PDAs come only with their own proprietary software. For example, some early Palm OS PDAs came only with Palm Desktop while later Palms such as the Treo 650 has the built-in ability to sync to Palm Desktop and/or Microsoft Outlook, while Microsoft's ActiveSync and Windows Mobile Device Center only synchronize with Microsoft Outlook or a Microsoft Exchange server.

Third-party synchronization software is also available for many PDAs from companies like Intellisync and Companion Link. This software synchronizes these handhelds to other personal information managers which are not supported by the PDA manufacturers, such as Goldmine and IBM Lotus Notes.

\section{SQL SERVER 2005 COMPACT EDITION}

Server 2005 Compact Edition (SSCE) provides a powerful yet lightweight data storage engine for building a variety of application types. SSCE is a lightweight $(<2 \mathrm{MB})$, free relational database engine that can be installed on any current Windows operating system. Because SSCE is the primary focus of this white paper, the full feature set is described in a later section. At a high level, SSCE supports tables, relations, constraints, complex query processing, transactions, replication, and data security. To program against SSCE, you use an ADO.NET managed provider with data access coding patterns similar to what you use for other managed providers, such as the SQL Server SQL Client managed provider. You can also access SSCE from unmanaged clients by using OLE DB. SSCE runs in-process as a set of libraries referenced by the using application, and is easy to deploy either with your application libraries or as a separate MSI install. SSCE can be easily deployed with Click Once applications or Xcopy-deployed onto mobile devices. SSCE will also be installed natively on Windows Mobile 6.0 or later.

The SSCE type system is a subset of the SQL Server 2005 type system, and does not support all features that a full SQL Server instance supports. Commonly used features from SQL Server for server applications that are not present in SSCE include stored procedures, triggers, views, functions, user defined data types, and the ability to participate in SQL Server Service Broker messaging. 
The core capability of SSCE is to allow secure transactional relational data access and storage. You can execute SQL queries that include Data Definition Language (DDL) and Data Manipulation Language (DML) queries through the SSCE engine. With SSCE, you create a database instance as a single .sdf file. Within that database you can define tables with primary keys and constraints. SSCE supports full referential integrity through foreign key constraints, and cascading deletes and updates. SSCE supports the following features:

Multiple concurrent connections for multi-threaded data access. Password protection and 128-bit encryption of the .sdf SSCE data file. A wide range of column data types. Scrollable, updateable cursors for fast and easy connected-data access. Databases that can grow up to $4 \mathrm{~GB}$ in size. Synchronization with SQL Server through merge replication and Remote Data Access (RDA).SSCE can now be run on any supported Windows operating system, including mobile devices, Tablet PCs, portable computers, desktops, and servers.

SSCE can be deployed by using Click Once.

When SSCE is used as a local data cache for a client or middle-tier application in distributed application architecture, you usually need to be able to synchronize the SSCE database with a back-end database server. You may need to populate the SSCE database tables initially from a back-end database, and you may also need to be able to push updated or new records from the client to the server database.

With SSCE, you have a number of options for synchronization. There are two built-in synchronization options: merge replication and RDA. Both of these capabilities allow you to synchronize data in both directions: from an SSCE database to a SQL Server 2005,. or SQL Server 2000 database over HTTP. Besides these capabilities, you could implement a custom synchronization solution by making calls to your own exposed Web service endpoints, allowing you to pass the data through custom business logic layers in both directions on the server side. Also, coming in the near future with the next release of Visual Studio (code named Orcas), there will be a new synchronization subsystem called the Occasionally Connected Systems (OCS) synchronization framework.

Merge replication is the most powerful built-in synchronization option because it allows the autonomous and independent updating of records on both the client (SSCE) and server (SQL Server) databases at the same time. SSCE participates in merge replication as a subscriber, and can subscribe to merge replication publications exposed from either SQL Server 2000 or SQL Server 2005. Merge replication handles many clients easily, because it also supports conflict detection and resolution on the server side. Merge replication is a little more complicated to set up, and requires specific database design features and configuration on the server side.RDA is the easiest built-in synchronization option to use. With RDA, there are no specific requirements on the server-side database. To synchronize data with RDA, you pull an entire result set into the SSCE database from the server side to initialize a table with the current data from the server. You can then push changes (inserts, updates, and deletions) back to the server when you choose to synchronize. To obtain changes that were made on the server side since the data was initially pulled, you have to pull the same result set again (after pushing your changes so that the pulled data contains your changes). RDA does not have any concurrency conflict detection or resolution. So, if you use it with an application on which multiple clients can modify different records at the same time, the last client to write changes to a record could overwrite recent changes by another client (last-in-wins). The OCS synchronization framework, when released, will allow you to choose between database-todatabase synchronization capabilities similar to both RDA and merge replication, or a more service-oriented approach in which you plug your own service endpoints into the synchronization pipeline. The former approach is simpler to set up because it requires the least custom code. The latter approach is a little more complex but enables you to insert business logic between the client end and the server, and also enables you to target other data sources that could be wrapped in an appropriate synchronization service.

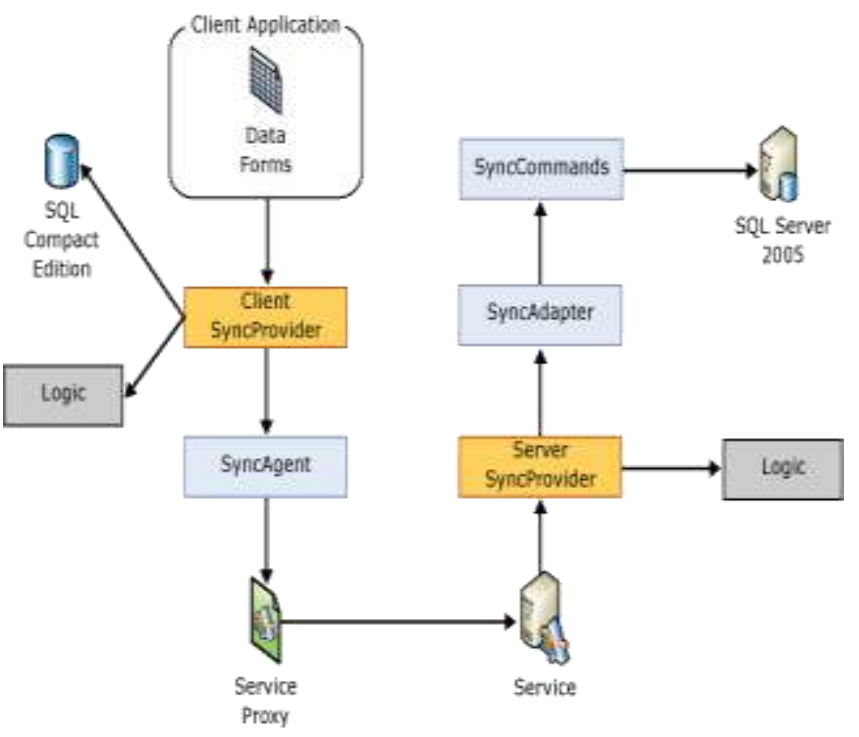

Figure OCS Synchronization Structure

\section{SYSTEM DESCRIPTION}

The Census Management System has 4 modules.1.Login Module. 2.Administrator Module. 3. Form registration Module. 4.Database Module

Login Module:-The login module is to create authentication for the user that is the employee who has authority to change or fill in the census form. The user has to provide the username and password in order to access the census form. The login module is the first module in our project. In the login module the user name and password is checked, this is the authorization part that is included in the module. A particular user can login for that particular session only; user cannot start from the middle of the application. The enumerator password and username is authorized by the administrator. The enumerator also acts as the database administrator for the census database

Administrator Module:-The administrator is the one who has privilege to create or delete a user account of an employee. The administrator is the one who fills or changes the census form. The administrator is the superior of this management system. The administrator is responsible for all the activities that is done in the PDA.As mentioned earlier the administrator is the one who authorises the enumerator. The administrator views the census report and can also access all the information. The administrator can edit or delete census data from the census database. The administrator can also view the census report, job analysis report etc. 
Registration Module:-The authenticated user after logging in has to fill in the forms with the specific details of a family. The registration module contains a unique identity number which is automatically generated whenever an enumerator starts to fill a new census form. As the unique identity is generated for each form the members in the household are also allocated a unique value. The registration module includes details like type of house owned, type of vehicle owned, no. of persons in the household, the monthly income of the family, working and non working, educational qualification etc. The registration module provides information about the census household, reports are produced based on the entered data. The unique identity number that is generated is useful for retrieving data whenever needed.

Database Module:-The database consists of the following tables in order to maintain this management system.

\section{Login table, User account table and Census report table.}

The database module is used to store the census data. Updating the database is done once the data is collected in the PDA. The database provides the back up for census data whenever needed. The login table and user account table contains the fields username, password and category. The census report table contains the registration form fields like identity number field, name, income, house, water connection, marital status, job type, illiterate or illiterate etc.

\section{ARCHITECTURAL DESIGN}

\section{Proposed System Overview}

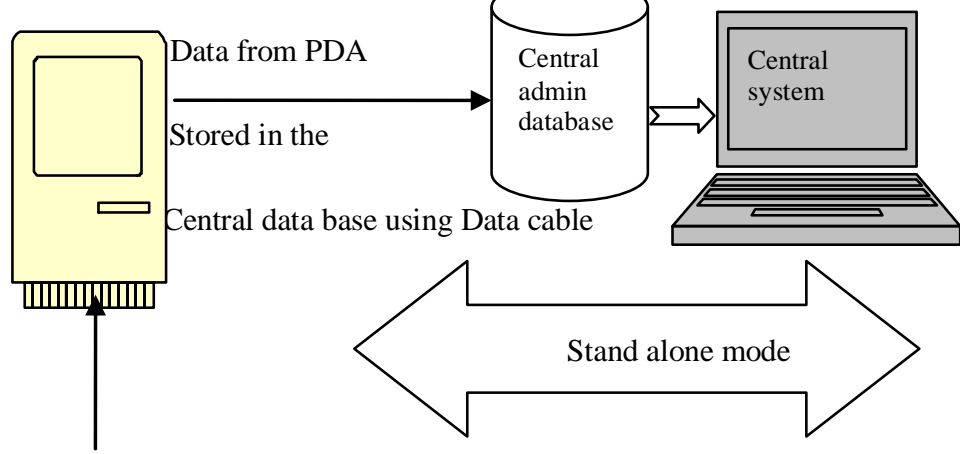

Enter census data into

PDA Enumerator

\section{Figure 4.1 Proposed System Overview}

The above diagram gives an overview of the process that is involved in census collection. The enumerator enters the data in the PDA and then the collected data is transferred to the central database system.

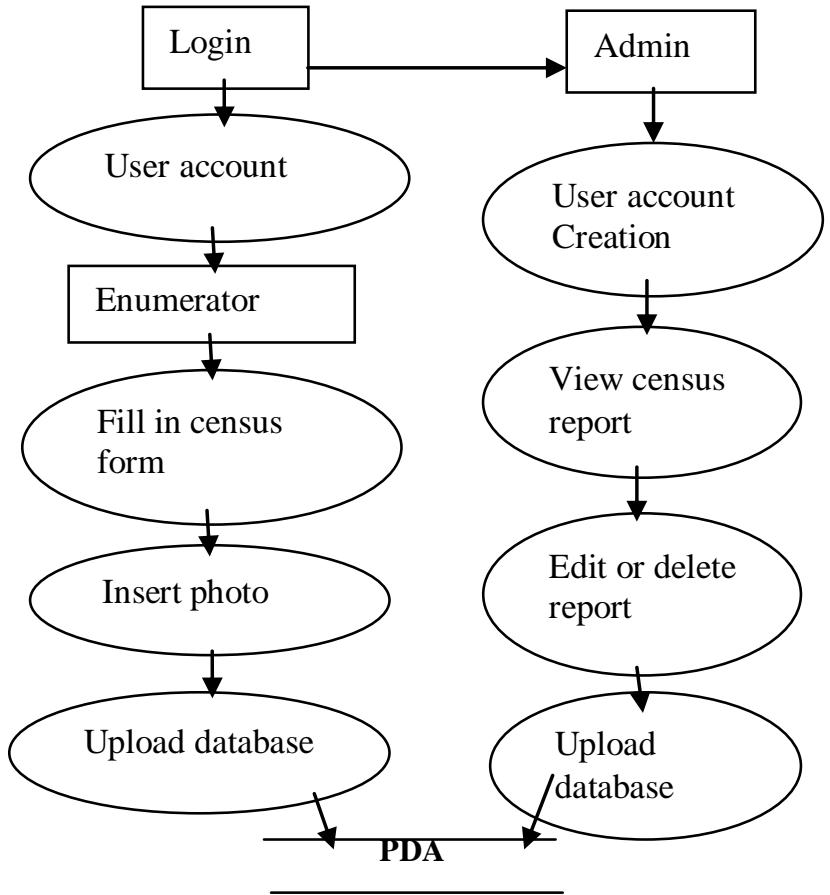

Figure 4.2 Data flow diagram

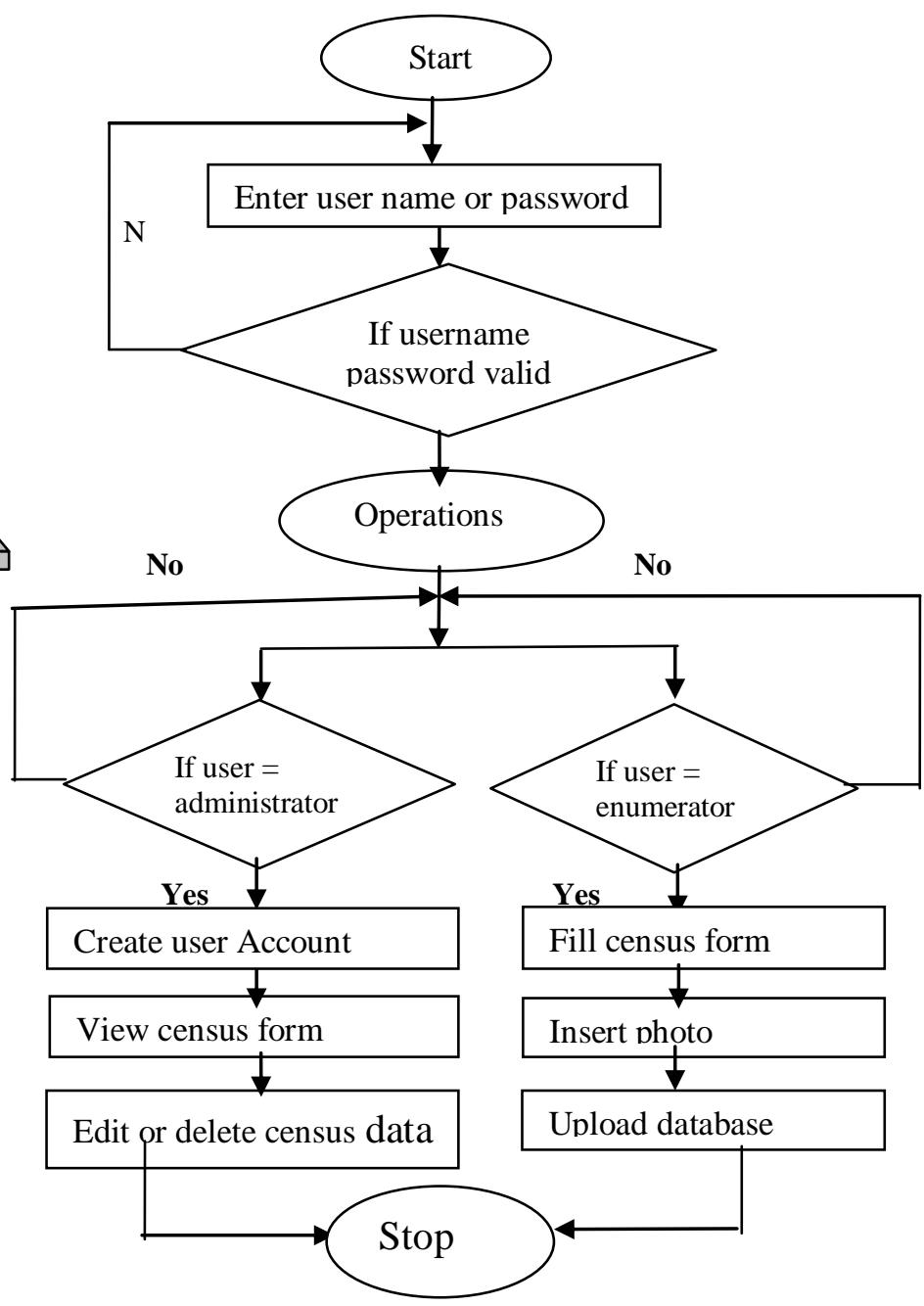

Figure 4.3 Basic Flow Chart 


\section{CONCLUSION}

Thus our paper "census management with PDA" has proven that it is easy to enumerate and present the census data meticulously with minimum hardship. In vast country like India, we need a system like this so that we can save time minimize expenditure and yet have an accurate data. This year preparations are being made for the taking $15^{\text {th }}$ census of our, new methods like biometrics which includes fingerprints and photographs are being introduced. In the 2011 census the national population register (NPR) is being brought out, our paper will be very helpful for maintaining the register.

\section{FUTURE ENHANCEMENT}

Now that we have done our application in a standalone mode, we can extend this to wireless mode. PDA's are coming with higher technological specifications like GPRS facility etc. We can easily extend our scope of the application to wireless mode which would be more useful. Fingerprints and retinal authorization can also be included when higher computational PDAs are used.

\section{REFERENCES}

Volume 9- No.9, November 2010

1. Automated Census System for Densely Inhabited Districts; Tadashi Ooishi, Takashi Nanaumi, Yoshiyuki Takei and Akihito Yamauci.

2. Instructions and Guidelines to enumerators to collect census manuals (Government of India).

3. Usability Study on the use Handheld Devices to Collect Census Data; Erica L.Olmsted.

4. www.censusindia.gov.in 